\title{
Perspectives on the Psychosocial Management of Oromandibular Dystonia
}

\author{
Allyson D. Page, Ph.D. ${ }^{1}$ and Lauren Siegel, M.Sc. ${ }^{1}$
}

\section{ABSTRACT}

Learning Outcomes: As a result of this activity, the reader will be able to (1) summarize common medical management approaches for treating individuals with oromandibular dystonia; (2) summarize the current assessment tools and instruments used to assess communicative participation and quality of life in individuals with dysarthria; and (3) describe how patient-reported outcome measures can impact and inform clinical practice in this clinical population.

Oromandibular dystonia (OMD) is a disorder of movement characterized by tonic muscle contractions that can result in involuntary, repetitive, and patterned muscle contrac- tions of the lingual musculature, labial musculature, and/or muscles of mastication. ${ }^{1}$ In 1899, Gowers first described OMD as a condition producing clonic and tonic jaw
${ }^{1}$ School of Communication Sciences and Disorders, Elborn College, Western University, London, Ontario, Canada.

Address for correspondence: Allyson Page, Ph.D., School of Communication Sciences and Disorders, Elborn College, 1201 Western Road, Western University, London, Ontario Canada, N6G 1H1 (e-mail: allyson.page@uwo.ca).
Innovations in Dysarthria Management; Guest Editor, Kathryn Yorkston, Ph.D.

Semin Speech Lang 2017;38:173-183. Copyright (C) 2017 by Thieme Medical Publishers, Inc., 333 Seventh Avenue, New York, NY 10001, USA. Tel: +1(212) 5844662.

DOI: https://doi.org/10.1055/s-0037-1602836.

ISSN 0734-0478. 
contractions. ${ }^{2}$ Eleven years later, in 1910, Meige reported blepharospasm with associated jaw, lingual, and pharyngeal contractions. ${ }^{3}$ This combination of blepharospasm and OMD is now called Meige syndrome. Consequently, OMD is variable in its presentation and it is classified based on location of the dystonic activity. This classification includes jaw opening, jaw closing, jaw deviation, jaw retraction, labial dystonia, lingual dystonia or any combination of these. ${ }^{4}$ Due to the varying clinical presentations of OMD and ranges of severity, OMD can be very difficult to diagnose and to treat. ${ }^{5,6}$ Furthermore, the rarity of OMD can make this dystonia difficult to diagnose and can result in misdiagnosis or delayed diagnosis. ${ }^{7}$ Patients often have to consult several physicians before their dystonia is correctly identified. ${ }^{8}$ Common misdiagnoses include temporomandibular joint syndrome, myasthenia gravis, dental malocclusion, and edentulous movements. ${ }^{8}$

OMD can be further classified as either primary or secondary in nature. Dystonia is classified as primary if it occurs in the absence of other clinical symptoms, or if it is inherited. Dystonia is classified as secondary when it occurs in association with another known disease. ${ }^{8}$ OMD is considered a focal dystonia, meaning that the dystonia affects a single body region. Dystonia, however, can also present as segmental (affecting more than one adjacent body region), multifocal (affecting more than one nonadjacent body region), hemifocal (affecting the muscles of only one side of the body), or generalized (affecting multiple muscle regions throughout the body). ${ }^{9}$

$\mathrm{OMD}$ is a rare condition, and the exact number of cases is largely unknown. The incidence of OMD has been estimated at 3.3 cases per million persons and the prevalence is estimated at 68.9 cases per million persons. ${ }^{10}$ The average age of onset of $\mathrm{OMD}$ is estimated to be 66 years (range 40 to 86 years) with more women affected than men by a ratio of $4: 1{ }^{10}$ Although it appears that most cases of OMD are sporadic in nature, evidence supporting a possible genetic cause for OMD was suggested by Steinberger and colleagues who reported a mutation of the $\mathrm{GCH} 1$ gene located on chromosome 14. ${ }^{6,11}$

The etiology of OMD is largely unknown and it is often idiopathic in nature. ${ }^{6}$ However, possible etiologic factors identified can include hypoxia, metabolic disorders, neuroleptic exposure, central nervous system or head trauma, diencephalitic stroke, demyelinating lesions in the upper brain stem, or neurodegenerative diseases such as Parkinson's disease. ${ }^{4,12}$ Druginduced dystonia may be caused by levodopa, dopamine agonists, antipsychotic drugs, anticonvulsant agents, serotonin-reuptake inhibitors, and rarely by other miscellaneous drugs. ${ }^{8}$ Exposure to certain chemicals such as manganese, carbon monoxide, or carbon disulfide may also result in dystonia. ${ }^{8}$ The pathophysiological underpinning of OMD is thought to be derived from dysfunction of the basal ganglia, specifically dysfunction of the basal ganglia-thalamocortical circuits. ${ }^{13}$ In addition, a neurochemical imbalance in cholinergic and dopaminergic activity has been suggested in OMD. ${ }^{12}$ Although OMD is considered primarily a disorder of motor control, a sensory impairment has also be suggested including, impaired inhibitory integration of somatosensory inputs at the cortical, subcortical, and spinal levels. ${ }^{13}$ A sensory component to OMD is also evidenced in sensory tricks or geste antagoniste. These are specific movements or actions an individual with dystonia can make to temporarily inhibit dystonic postures or movements. Sensory tricks can be helpful in reducing dystonic contractions in some individuals with OMD. ${ }^{9}$ Examples include touching the hand to the chin or jaw to achieve jaw closure or touching the hand to the lips to relieve jaw spasms, singing, or humming. Sleeping and relaxation have also been reported to improve dystonic symptoms. ${ }^{6}$

\section{CONSEQUENCES OF OROMANDIBULAR DYSTONIA}

The dystonic muscular contractions associated with OMD can have devastating consequences on the daily functioning of those affected. These dystonic movements commonly result in involuntary clenching, opening, or deviation of the jaw, as well as abnormal posturing of the lingual or labial musculature. 8 The altered orofacial aesthetics associated with OMD, such as jaw deviation and involuntary tongue protrusion, can lead to feelings of embarrassment and reduced self-confidence. ${ }^{14} \mathrm{OMD}$ has 
been linked to depression and a reduction in quality of life (QoL). ${ }^{7}$ Severe cases may cause jaw pain, difficulty chewing, dysphagia, and dental trauma. ${ }^{8}$ A study by Papapetropoulos and Singer found that dystonic eating dysfunction may complicate OMD, leading to weight loss. ${ }^{15}$ In a cohort of 53 patients with primary and secondary OMD, there was a $15.6 \%$ prevalence of eating dysfunction. Over half of the patients with eating dysfunction also reported significant weight loss. Eating dysfunction associated with OMD may include pain during eating and further social embarrassment when accompanied by drooling or choking. ${ }^{15}$ OMD may also cause oral trauma. Jaw-closing dystonia most often results in trauma, consisting of persistent grinding of the teeth or biting of the tongue, wearing of the enamel or early loss of teeth, trauma of the lips or gums, or persistent jaw pain. ${ }^{7}$ Dystonic activity may also cause head and jaw pain, muscular tension or tiredness, and a reduced salivary flow rate. ${ }^{7}$ Involuntary closing of the jaw may produce inappropriate deviation of the mandible, partial dislocation, soft tissue trauma, and bone resorption. ${ }^{16}$

In addition to altered orofacial esthetics, orofacial pain, difficulty with chewing, and dental trauma, OMD can also result in a slow hyperkinetic dysarthria. ${ }^{17,18}$ Darley and colleagues presumed the neuromuscular deficits associated with the dysarthria of dystonia to cause slow, involuntary movements, with irregular rhythm, reduced range, and excessive tone. ${ }^{18}$ They found the most deviant speech dimensions of dystonia to be imprecise consonant articulation, vowel distortion, harsh voice quality, irregular articulatory breakdown, strainedstrangled voice quality, monopitch, monoloudness, vocal tremor, alternating loudness, and voice stoppages. ${ }^{18}$ In this original classification of dystonia, spasmodic dysphonia (hyperadduction of abductor or adductor laryngeal muscles) was included in the description of dystonia. Therefore, the laryngeal impairments described in the Mayo Clinic description of dystonia, such as a harsh voice, strained-strangled vocal quality, voice stoppages, and vocal tremor, should not be included in the perceptual features of the dysarthria associated with OMD. ${ }^{18}$ Interestingly, Kreisler et al described a dysarthric profile characterized by imprecise consonant production and a rough voice, with changes in intensity, and hypernasality despite variability in speech production across 14 patients with OMD. ${ }^{19}$ Dykstra and colleagues studied the speech intelligibility of an individual with focal lingual dystonia. ${ }^{20}$ The results of this acoustic and perceptual analysis of intelligibility revealed that intelligibility deficits were associated with palatal fricatives being perceived as more fronted alveolar fricatives (i.e., $/ \int \rightarrow \mathrm{s} /$ ). Similarly, Golper and colleagues described a single patient with severe lingual dystonia and a severe speech intelligibility deficit as having difficulty with forward tongue postures. ${ }^{21}$ For example, the vowel /a/ was fronted, raised and diphthongized to become /aI/. It appears that abnormal lingual protrusion and elevation may be a contributor to the intelligibility deficits observed in individuals with focal lingual dystonia, or OMD affecting the tongue. Weismer and colleagues suggest that the tongue is the most influential articulator in speech intelligibility. ${ }^{22}$ Weismer and colleagues also assert that tongue control may be more strongly related to speech intelligibility in individuals with neuromotor pathology than lip/jaw control. Continued study of the perceptual and acoustic correlates of impaired speech production in OMD is warranted.

\section{MEDICAL MANAGEMENT}

Because there is no known cure for OMD, the primary goals of therapeutic management focus on various treatments and strategies aimed at reducing dystonic symptoms, improving orofacial aesthetics, as well as improving masticatory capabilities, swallowing function, and speech production. The literature reviewing traditional and contemporary therapeutic options for the management of OMD cite pharmacotherapy, dental appliances (i.e., bite block therapy), chemodenervation, and neurosurgery as potential treatment options.

\section{Pharmacologic Treatment}

Systemic pharmacologic agents used to treat OMD include anticholinergics (e.g., trihexyphenidyl, benztropine), benzodiazepines (e.g., clonazepam, lorazepam, diazepam), baclofen, 
and medications such as tetrabenazine, which depletes dopamine and blocks dopamine receptors. ${ }^{6,23}$ Although these drugs have been found useful in some patients, it is not the preferred form of treatment for focal dystonias because there is only modest improvement and frequent side effects. ${ }^{23}$ Medications are most effective in cases of widespread dystonia. One of the besttolerated medications for treating OMD is clozapine. $^{24}$

\section{Dental Appliances}

A device for treating OMD is a bite block. A bite block is a device that is tailored to the individual patient; it is placed between the upper and lower teeth to help with jaw stability and positioning. Bite block therapy has been shown to improve facial appearance, articulatory precision, and hyperactive movements observed in OMD. ${ }^{12}$ Dworkin found that insertion of a bite block in two individuals with OMD secondary to Meige syndrome resulted in immediate improvement in orofacial postural control and speech intelligibility. ${ }^{12}$ Both individuals demonstrated near normal speech intelligibility when wearing a bite block. It was hypothesized that the bite block neutralized dystonic activity by facilitating stabilization of the mandible, which helped to diminish dystonic jaw movements. Dworkin also suggested that stabilizing the mandible helps to reduce the disruptive effects of dystonic jaw movements on normal labial and lingual function. A bite block may prevent enamel wear, decrease the load on oromandibular joints, and improve chewing function. ${ }^{25}$ In addition to stabilizing the jaw and reducing dystonic movement, a bite block may provide sensory information for the correct placement of other articulators. ${ }^{14}$ Although these techniques are not long-lasting, they are noninvasive and may aid other forms of therapy.

\section{Chemodenervation}

The most contemporary and well-tolerated treatment for OMD is achieved via localized injection of botulinum toxin into the affected muscle(s). ${ }^{26}$ Botulinum toxin was introduced as a therapeutic agent for OMD in $1977 .^{27}$
Botulinum toxin is a protein produced by the anaerobic bacterium Clostridium botulinum. ${ }^{28}$ There are seven antigenically distinct serotypes, but only types $\mathrm{A}, \mathrm{B}$, and $\mathrm{E}$ have been used in humans. Botulinum toxin type A (BoNT-A) is the most effective serotype in the treatment of OMD. ${ }^{29}$ There are currently three Food and Drug Administration-approved BoNT-A products available: onabotulinumtoxinA (Botox, Allergan, Inc., Irvine, CA), abobotulinumtoxinA (Dysport, Ipsen Biopharmaceuticals, Ltd., Slough, UK), and incobotulinumtoxinA (Xeomin, Merz Pharmaceuticals, Raleigh, $\mathrm{NC}) .^{29}$ Botulinum toxin works by blocking the presynaptic release of acetylcholine into the neuromuscular junction and thereby producing a temporary paralysis of the injected muscle. ${ }^{28}$ After binding to presynaptic cholinergic nerve terminals, BoNT-A decreases the frequency of acetylcholine release. The flaccid paralysis/weakness induced by BoNT-A does not appear for 1 to 3 days; however, by 2 weeks, a marked effect is present and the effects last for approximately 3 months. $^{27}$

Botulinum toxin is injected locally into the dystonic muscle(s). Dosing requires an individualized approach because it is based on the mass of the muscle being injected and individual characteristics of the patient such as body mass and any pre-existing weakness. ${ }^{26}$ In jawopening dystonia, the submental and/or lateral pterygoid muscles are injected, and in jawclosing dystonia, the temporalis and masseter muscles are injected. Lingual dystonia has been treated with injections to the genioglossus, hypoglossus, and intrinsic muscles of the tongue. ${ }^{20,23}$ Jankovic and colleagues reported that injection of BoNT-A into the masseter, temporalis, and lateral pterygoid muscles results in reduction of spasm and improvement in chewing and speech, in $\sim 70 \%$ of patients with OMD. ${ }^{30}$ Electromyography (EMG) can be used to determine the proper injection site(s). EMG provides information about muscles demonstrating increased activity associated with a dystonic contraction. ${ }^{23}$ In the case of $\mathrm{OMD}$, it is difficult to determine all the muscles involved because EMG recordings are not completely accurate and the pattern of muscle involvement may change over time. ${ }^{23}$ For this reason, injection site and dose may vary for each 
appointment. Side effects associated with BoNT-A are normally tolerated well but can include mild dysarthria, masticatory difficulties, and mild dysphagia. ${ }^{26}$

\section{Neurosurgery}

A less common management option for OMD is neurosurgery. There is a modest literature exploring the potential benefit of deep brain stimulation (DBS) of the globus pallidus internus for those with focal dystonias such as Meige syndrome. ${ }^{31}$ For example, Capelle and colleagues reported improvements in dystonic movements in an individual with Meige syndrome following bilateral pallidal DBS surgery, but only a minimal improvement in swallowing function. ${ }^{31}$ Foote and colleagues demonstrated improvements in dystonic symptoms following right unilateral globus pallidus internus DBS, with a gradual return of symptoms 6 months postoperatively. ${ }^{32}$ Despite a limited empirical literature, further exploration of neurosurgical techniques to manage severe, medication-resistant forms of focal dystonias is warranted.

\section{MANAGEMENT OF PSYCHOSOCIAL ISSUES}

Unfortunately, little attention has been given to the impact of the various medical treatment approaches on speech production. Some empirical literature suggests improvements to articulatory precision following the use of a bite block, and preliminary evidence is available to suggest that, depending on the type and location of OMD, there may be an improvement to speech intelligibility following BoNT-A injections. ${ }^{12,20,33}$ Similarly, few studies examine the impact of OMD on psychosocial aspects such as communicative participation and QoL as it relates to communication. This is unfortunate because dysarthria can be a disabling aspect of OMD and can have an adverse impact on psychosocial functioning. ${ }^{20,33,34}$ In the section that follows, we introduce and describe patientreported outcome measures that assess two aspects of psychosocial functioning: communicative participation and QoL. We describe the current state of knowledge as it relates to communicative participation and QoL in this clinical population, and finally, we will advocate that speech-language pathologists (SLPs) have a role in the care of individuals with OMD through the inclusion of patient-reported outcome measures to provide a comprehensive and holistic management plan.

Historically, clinical focus has taken an impairment-based perspective to treating communication disorders rather than managing a communication disorder that includes psychosocial outcomes. ${ }^{35}$ Recently, there has been a shift in attention to the psychosocial aspects of health across the field of health care in general as well as in the field of communication disorders. We would be remiss to ignore the profound social, emotional, and vocational consequences of OMD. Decreased speech intelligibility, difficulty managing oral intake of food and liquids, dysphagia, and altered orofacial aesthetics are likely sequelae of a diagnosis of OMD. Because treatment aims to manage symptomatology versus being curative, many individuals with OMD may feel a sense of helplessness over their condition. This can result in feeling depressed and socially isolated. ${ }^{20}$ Unfortunately, SLPs may feel ill equipped to manage OMD, or SLPs may feel that they do not have a role in the management of the individual with OMD. This may be due to the rarity of the disease and therefore the lack of resources and exposure to this unique patient population, or because the main treatment approach is achieved medically via botulinum toxin injections. In fact, SLPs can play a significant role in the management of this clinical population via a psychosocial management approach, specifically, the impact of OMD on communicative participation and QoL.

The construct "communicative participation" is derived from the World Health Organization's International Classification of Functioning, Disability, and Health (ICF). ${ }^{36}$ The ICF provides a conceptual framework of disability that supports the focus on the psychosocial consequences of OMD. The ICF asserts "Health is a state of complete physical, mental and social well-being, and not merely the absence of disease or infirmity." ${ }^{36}$ The ICF defines impairment as a "problem in body function or body structure," activity as the 
"execution of a task or action by an individual," and participation as "involvement in life situations." 36 The ICF framework also includes environmental and personal contextual factors that contribute to the consequences of a health condition. Eadie and colleagues extended the ICF definition of participation to communication by defining communicative participation as "taking part in life situations where knowledge, information, ideas or feelings are exchanged." 37 One component of communicative participation called communicative effectiveness was defined by Hustad as a person's ability to successfully communicate messages in home and community settings to fulfill life roles. ${ }^{38}$ Communicative participation encompasses many life situations including personal care, household management, leisure, learning, employment, relationships, and community life. ${ }^{37,39}$

In addition to communicative participation, obtaining patient reports of $\mathrm{QoL}$ can provide important information about the impact of OMD, from the perspective of the individual. The World Health Organization defines health, as it relates to QoL as a "state of complete physical, mental, and social wellbeing and not merely the absence of disease." ${ }^{40}$ Another, less studied aspect of QoL is quality as it relates specifically to communication. The American Speech-Language Hearing Association (ASHA) defines quality of communication life as "the extent to which a person's communication acts, influenced by personal and environmental factors, and filtered through a person's own perspective allow for meaningful participation in life situations." ${ }^{\text {11 }}$ Measuring communicative participation and communication-related QoL is important because it advances our understanding of both the impact of disablement and the contextual factors that affect it, and it helps to develop and revise models of intervention. ${ }^{37}$

Although the ICF provides a theoretical framework, research is also beginning to examine the psychosocial consequences of OMD. The experience of a speech disorder is highly individualized and dependent on a wide array of personal, contextual, and environmental factors. Qualitative research methods can be used for studying the complex nature of speech disorders because they identify the individual with the disability as the "expert," rather than the researcher or clinician. ${ }^{42}$ A qualitative approach provides space for "participant voice," which allows for a genuine account of the insider's experience. Siegel explored the consequences of $\mathrm{OMD}$ on communicative participation from the insider's perspective. ${ }^{43}$ Eight individuals with OMD and dysarthria participated in face-to-face interviews. Three major themes emerged from this study. The first theme, "speaking is different now," contained information about the physical effort required to speak with OMD. Participants described changes in their speech production such as increased physical effort, slowed rate of speech, and difficulty articulating certain speech sounds. Participants explained how changes in speech production affected their communicative participation in everyday life. They reported unpredictability of when they may or may not be understood by others, which resulted in less frequent communicative participation, especially in high-stress/unfamiliar situations. The second major theme, "my roles have changed," addressed changes in participants' everyday lives since their diagnosis. Participants described how OMD impacted their roles in the workplace and at home, as well as in social activities. Finally, the third major theme, "I accept it and move on," reflected how participants were able to carry on with their lives after being diagnosed with OMD. Participants explained strategies that they found useful including support from family and friends, educating others, alternative activities, and using humor. Overall, the study by Siegel and colleagues illustrates the functional barriers and restrictions to communicative participation experienced by individuals with OMD and the resulting interferences to communicative participation they may cause. This study also demonstrates that consequences of OMD extend beyond the speech impairment.

Dykstra (Page) and her colleagues have explored communicative participation in individuals with OMD using patient-reported questionnaires. ${ }^{20,33,34}$ Across all studies, individuals with $\mathrm{OMD}$ have reported significant reductions in participation as compared with healthy control participants. The Voice Activity 
and Participation Profile (VAPP) was administered to an individual with focal lingual dystonia in a 2007 case study by Dykstra and colleagues. $^{20}$ The VAPP is a 28 -item questionnaire developed to evaluate perceptions of speech problems, activity limitations, and participation restrictions. ${ }^{44}$ There were significant reductions reported on all domains of the VAPP (e.g., self-perceived severity, effect on job, effect on daily communication, effect on social communication, and effect on emotion) as a result of OMD. Although a preliminary case report, this study was an important starting point for examining the importance of the impact of OMD on an individual's daily functions in the context of their personal, social, and environmental milieu. ${ }^{20,44}$ This study also revealed that only a mild to moderate reduction in speech intelligibility (95\% sentence intelligibility, $85 \%$ single-word intelligibility, based on the Speech Intelligibility Test ${ }^{45}$ ) significantly and negatively impacted this individual's participation. ${ }^{20}$ For example, the participant experienced loss of employment and reported feelings of embarrassment, self-esteem issues, and self-image issues and communicative and social avoidance as a result of her dysarthria. More recently, in 2015, Dykstra and colleagues examined self-rated communicative effectiveness in 10 speakers with OMD and 10 healthy control participants. Using the Communicative Effectiveness Survey (CES), ${ }^{46}$ speakers with OMD reported reduced effectiveness conversing with a stranger or a familiar person on the telephone, having a conversation with a family member or friend at home, having a conversation while traveling in a car, and participating in conversation with strangers in a quiet place. ${ }^{33}$ Despite having milder intelligibility deficits (sentence intelligibility: 90.91\%, Speech Intelligibility $\mathrm{Test}^{4}$ ), self-perceived reductions in communicative effectiveness were reported as barriers across a range of communicative contexts and situations. There is a growing literature demonstrating that restrictions to participation are not necessarily predicted or dependent on the severity of the speech intelligibility deficit. ${ }^{20,33,47}$ Therefore, the psychosocial impact of impaired communication does not necessarily correlate strongly with traditional clinical measurement.
There appears to be only one published study, by Page and colleagues, that has sought to explore communication-related $\mathrm{QoL}$ of individuals with OMD. ${ }^{34}$ One aspect of this study evaluated how 10 individuals with OMD self-rated communication-related QoL as compared with control participants. Using the ASHA Quality of Communication Life scale (QCL), results showed significant differences between individuals with OMD and control participants across all subdomains and domains of the QCL. For example, individuals with OMD reported reductions in $\mathrm{QoL}$ related to socialization and activities, confidence and selfconcept, roles and responsibilities, and overall QoL. ${ }^{34}$ These results demonstrate that OMD can have a pervasive impact across all aspects of QoL as it relates to communication.

The examination of health-related QoL of individuals with OMD has received more attention than communicative participation or communication-related QoL, but the literature is still quite limited. The majority of these published studies have evaluated health-related QoL before and after botulinum toxin injections to determine if this medical intervention is effective for improving QoL. ${ }^{1,48,49}$ Most of these studies have used the Glasgow Benefit Inventory (GBI) to measure the change in a patient's health produced by an intervention. The GBI is an 18-item questionnaire, divided into three domains (general subscore, social support subscore, physical health subscore). Overall, these studies demonstrated improvements in health-related QoL following botulinum toxin injections across all domains of the GBI. ${ }^{1,48}$ Unfortunately, the GBI does not address impaired speech production or the impact of dysarthria. Natasi and colleagues published one of the first studies to examine health-related $\mathrm{QoL}$ using an OMD-specific health-related QoL instrument called the Oromandibular Dystonia Questionnaire (OMDQ25). ${ }^{5,49}$ The OMDQ-25 is a 25 -item questionnaire divided into five subscales that evaluates psychosocial, cosmetic, speech, eating, as well as a "general" score that contributes to an overall health-related QoL score. Each item is rated on a 5-point scale with a range of descriptors relating to degree of impairment: never, seldom, sometimes, often, and always. Natasi 
et al used the OMDQ-25 to investigate the impact of BoNT-A injections on QoL for individuals with predominantly lingual dystonia. ${ }^{49}$ Overall, this study reported that BoNTA injections can result in a consistent and measureable improvement in QoL for individuals with predominantly lingual dystonia. Merz and colleagues also reported improvements across all subscales of the OMDQ-25 following BoNT-A injections, with the "cosmetic" subscale demonstrating the greatest improvement following injections with BoNTA. ${ }^{5}$ Taken together, these research studies advocate for comprehensive, patient-centered management of the individual with OMD, and they provide insight into the everyday consequences of living with OMD.

Other patient-reported measures with clinical utility are being developed. Recently, Baylor and colleagues developed the Communicative Participation Item Bank, 10-item short form (CPIB) ${ }^{50}$ The CPIB is a 10 -item, disorder-generic instrument developed for community-dwelling adults with communication disorders. The items contained on the CPIB are meant to provide a patient-reported assessment of the extent to which their communication disorder interferes with communicative participation across a variety of speaking situations including, but not limited to, talking on the telephone to get information, ordering a meal at a restaurant, and communicating when needing to say something quickly. Items on the $\mathrm{CPIB}$ are rated on a 4-point scale; a score of 3 represents that the communication disorder does not at all interfere with participating in a particular speaking situation, whereas a score of 0 represents that the communication disorder very much interferes with participating in a particular speaking situation. An advantage of the CPIB is that the summary score (ranging from 0 to 30) can be converted to item response theory theta values and/or standard $T$ scores $($ mean $=50$, standard deviation $=10)$.

The CES, designed by Donovan and colleagues, asks respondents to rate their effectiveness communicating in various social contexts and situations. ${ }^{46}$ The CES allows speakers with dysarthria and their communication partners to rate communicative effectiveness in various life situations to identify which situations are perceived as most difficult. ${ }^{38}$ The CES is an eight-item questionnaire focusing on communicative participation that is rated on a 4-point, Likert scale. A score of 1 represents that communication is not effective and a score of 4 represents that communication is very effective across various speaking situations such as having a conversation while traveling in a car, being part of a conversation in a noisy environment, and conversing at a distance, to name a few examples. Both the CPIB and CES can provide valuable patient-reported information that can assist in guiding treatment planning and comprehensive outcome measurement of a communication disorder.

Understanding QoL as it relates to communication has received limited attention in this population. Most research on QoL in OMD focuses on health-related outcomes. The impact of the communication disorder on QoL is either not part of the QoL instrument or it is addressed as a subcomponent of health-related QoL. There are some notable examples of instruments that evaluate QoL in communication disorders. The Voice-Related Quality of Life scale, ${ }^{51}$ the Voice Handicap Index ${ }^{52}$ the Quality of Life in the Dysarthric Speaker, ${ }^{53}$ and ASHA's QCL scale are some instruments designed specifically to evaluate QoL in communication disorders and should be considered if we are to provide a comprehensive approach to the management of OMD. ${ }^{41}$

The cumulative body of knowledge currently accumulated in our understanding of the psychosocial consequences of OMD demonstrates that psychosocial issues are pervasive, they are of concern to individuals with OMD, and they are important clinically. Fortunately, the recent shift in health care that acknowledges the importance of psychosocial aspects of health has resulted in an increased number of patient-reported outcome measurement tools available for use in both research and clinical settings. These measurement tools not only afford SLPs the opportunity to understand the consequences of OMD from the perspective of the individual, but can help to shape treatment approaches that target specifically communicative participation, contextual/situational barriers to successful communication, and ultimately inform best practices. 


\section{THE ROLE OF SPEECH-LANGUAGE PATHOLOGISTS IN MANGEMENT OF OROMANDIBULAR DYSTONIA}

A diagnosis of OMD is relatively rare, but the impact of this disorder can be profoundly disabling with impacts observed on healthrelated QoL, quality of communication life, and communicative participation. Although intervention is largely medically based and achieved via botulinum toxin injections, SLPs have a unique role in the management of their clients with this disorder. As communication specialists, we must advocate for the clients that we serve using a comprehensive approach to management. Although the traditional impairment-based outcome measures should not be abandoned, we cannot ignore the management of our clients through a psychosocial lens. This approach includes the assessment of communicative participation and QoL outcomes to provide comprehensive and holistic care. Using a combination of objective clinical measures and patient-reported outcome measures, we can begin to understand our client's perception of their communication disorder. This knowledge may provide prognostic information that can guide our care plan and it can help to inform meaningful clinical outcomes for our clients. This may be achieved through the provision of educational strategies and targeted support to this underserviced clinical population.

\section{FINANCIAL DISCLOSURE}

The authors report no financial interests or conflicts of interest.

\section{REFERENCES}

1. Teemul TA, Patel R, Kanatas A, Carter LM. Management of oromandibular dystonia with botulinum A toxin: a series of cases. Br J Oral Maxillofac Surg 2016;54(10):1080-1084

2. Gowers WR. A Manual of Diseases of the Nervous System. Vol. 2. 3rd ed. London : Churchill; 1899:200

3. Meige H. Les convulsions de la face, une forme clinique de convulsions facile, bilaterale et mediane. Rev Neurol (Paris) 1910;20:437-443

4. Tan EK, Jankovic J. Tardive and idiopathic oromandibular dystonia: a clinical comparison. J Neurol Neurosurg Psychiatry 2000;68(02):186-190
5. Merz RI, Deakin J, Hawthorne MR. Oromandibular dystonia questionnaire (OMDQ-25): a valid and reliable instrument for measuring health-related quality of life. Clin Otolaryngol 2010;35(05): 390-396

6. Tan EK. Oromandibular dystonia. In: Brin MF, Comella CL, Jankovic J, eds. Dystonia: Etiology, Clinical Features, and Treatment. Philadelphia, PA: Lippincott Williams \& Wilkins; 2004: 167-174

7. Bakke M, Larsen BM, Dalager T, Moller E. Oromandibular dystonia-functional and clinical characteristics: a report on 21 cases. Oral Surg Oral Med Oral Pathol Oral Radiol 2013;115(01): e21-e26

8. Tarsy D, Simon DK. Dystonia. N Engl J Med 2006;355(08):818-829

9. Brin MF, Comella CL. Pathophysiology of dystonia. In: Brin MF, Comella CL, Jankovic J, eds. Dystonia: Etiology, Clinical Features, and Treatment. Philadelphia, PA: Lippincott Williams \& Wilkins; 2004:5-10

10. Nutt JG, Muenter MD, Aronson A, Kurland LT, Melton LJ III. Epidemiology of focal and generalized dystonia in Rochester, Minnesota. Mov Disord 1988;3(03):188-194

11. Steinberger D, Topka H, Fischer D, Müller U. GCH1 mutation in a patient with adult-onset oromandibular dystonia. Neurology 1999;52(04): 877-879

12. Dworkin JP. Bite-block therapy for oromandibular dystonia. J Med Speech-Lang Pathol 1996;4(01): 47-56

13. Møller E, Bakke M, Dalager T, et al. Somatosensory input and oromandibular dystonia. Clin Neurol Neurosurg 2013;115(07):1141-1143

14. Lee KH. Oromandibular dystonia. Oral Surg Oral Med Oral Pathol Oral Radiol Endod 2007;104 (04):491-496

15. Papapetropoulos S, Singer C. Eating dysfunction associated with oromandibular dystonia: clinical characteristics and treatment considerations. Head Face Med 2006;2(47):47

16. Gandhi YR. Oro-mandibular dystonia. Natl J Maxillofac Surg 2010;1(02):150-152

17. Darley FL, Aronson AE, Brown JR. Differential diagnostic patterns of dysarthria. J Speech Hear Res 1969a;12(02):246-269

18. Darley FL, Aronson AE, Brown JR. Clusters of deviant speech dimensions in the dysarthrias. J Speech Hear Res 1969b;12(03):462-496

19. Kreisler A, Verpraet AC, Veit S, et al. Clinical characteristics of voice, speech, and swallowing disorders in oromandibular dystonia. J Speech Lang Hear Res 2016;59(05):940-949

20. Dykstra AD, Adams S, Jog M. The effect of botulinum toxin type $\mathrm{A}$ on speech intelligibility 
in lingual dystonia. J Med Speech-Lang Pathol 2007;15(02):172-186

21. Golper LA, Nutt JG, Rau MT, Coleman RO. Focal cranial dystonia. J Speech Hear Disord 1983; 48(02):128-134

22. Weismer G, Yunusova Y, Bunton K. Measures to evaluate the effects of DBS on speech production. J Neurolinguist 2012;25(04):74-94

23. Tinter R, Jankovich J. Botulinum toxin type A in the management of oromandibular dystonia and bruxism. In: Brin MF, Hallet M, Jankovich J, eds. Scientific and Therapeutic Aspects of Botulinum Toxin. Philadelphia, PA: Lippincott Williams \& Wilkins; 2002:1-12

24. Karp BI, Goldstein SR, Chen R, Samii A, Bara-Jimenez W, Hallett M. An open trial of clozapine for dystonia. Mov Disord 1999;14(04): 652-657

25. Blanchet PJ, Rompré PH, Lavigne GJ, Lamarche C. Oral dyskinesia: a clinical overview. Int J Prosthodont 2005;18(01):10-19

26. Münchau A, Bhatia KP. Uses of botulinum toxin injection in medicine today. BMJ 2000;320(7228): 161-165

27. Blitzer A, Sulica L. Botulinum toxin: basic science and clinical uses in otolaryngology. Laryngoscope 2001;111(02):218-226

28. Simpson LL. The origin, structure, and pharmacological activity of botulinum toxin. Pharmacol Rev 1981;33(03):155-188

29. Kazerooni R, Broadhead C. Cost-utility analysis of botulinum toxin type A products for the treatment of cervical dystonia. Am J Health Syst Pharm 2015; 72(04):301-307

30. Jankovic J, Schwartz K, Donovan DT. Botulinum toxin treatment of cranial-cervical dystonia, spasmodic dysphonia, other focal dystonias and hemifacial spasm. J Neurol Neurosurg Psychiatry 1990; 53(08):633-639

31. Capelle HH, Weigel R, Krauss JK. Bilateral pallidal stimulation for blepharospasm-oromandibular dystonia (Meige syndrome). Neurology 2003; 60(12):2017-2018

32. Foote KD, Sanchez JC, Okun MS. Staged deep brain stimulation for refractory craniofacial dystonia with blepharospasm: case report and physiology. Neurosurgery 2005;56(02):E415

33. Dykstra AD, Domingo Y, Adams SG, Jog M. Examining speech intelligibility and self- ratings of communicative effectiveness in speakers with oromandibular dystonia receiving botulinum toxin therapy. Canadian Journal of Speech Language Pathol Audiol 2015;39(04):334-345

34. Page AD, Siegel L, Jog M. Self-rated communication-related quality of life of individuals with oromandibular dystonia receiving botulinum toxin injections. Am J Speech Lang Pathol. 1-8. https://doi.org/10.1044/2017_AJSLP-16-0098
35. Eadie TL. The ICIDH-2: theoretical and clinical implications for speech-language pathology. J Speech Lang Pathol Audiol 2001;25:181-200

36. World Health Organization. ICF: International Classification of Functioning, Disability and Health. Geneva: Author; 2001

37. Eadie TL, Yorkston KM, Klasner ER, et al. Measuring communicative participation: a review of self-report instruments in speech-language $\mathrm{pa}^{-}$ thology. Am J Speech Lang Pathol 2006;15(04): 307-320

38. Hustad KC. Optimizing communicative effectiveness: bringing it together. In: Yorkston $\mathrm{K}$, Beukelman D, Strand EA, Bell KR, eds. Management of Motor Speech Disorders in Children and Adults. Austin, TX: Pro-Ed; 1999:483-537

39. Yorkston KM, Baylor CR, Dietz J, et al. Developing a scale of communicative participation: a cognitive interviewing study. Disabil Rehabil 2008;30(06):425-433

40. World Health Organization. ICIDH: International Classification of Impairments, Disability and Handicap. Geneva: Author; 1980

41. Paul D, Frattali C, Holland A, Thompson C, Caperton C, Slater S. Quality of Communication Life Scale (ASHA QCL). Rockville, MD: ASHA 2004

42. Dowling M. From Husserl to van Manen. A review of different phenomenological approaches. Int J Nurs Stud 2007;44(01):131-142

43. Siegel L. The Consequences of Oromandibular Dystonia on Communicative Participation: A Qualitative Study of the Insider's Experiences [master's thesis]. London: Western University; 2016

44. Ma EP, Yiu EM. Voice activity and participation profile: assessing the impact of voice disorders on daily activities. J Speech Lang Hear Res 2001; 44(03):511-524

45. Yorkston K, Beukelman D, Tice R. Speech Intelligibility Test. Lincoln, NE: Institute for Rehabilitation Science and Engineering at Madonna Rehabilitation Hospital; 2011

46. Donovan NJ, Velozo CA, Rosenbek JC. The Communicative Effectiveness Survey: investigating its item-level psychometric properties. J Med Speech-Lang Pathol 2007;15:433-447

47. Yorkston KM, Klasner ER, Swanson KM. Communication in context: a qualitative study of the experiences of individuals with multiple sclerosis. Am J Speech Lang Pathol 2001;10(02):126-137

48. Charous SJ, Comella CL, Fan W. Jaw-opening dystonia: quality of life after botulinum toxin injections. Ear Nose Throat J 2011;90(02):E9-E12

49. Nastasi L, Mostile G, Nicoletti A, Zappia M, Reggio E, Catania S. Effect of botulinum toxin treatment on quality of life in patients with isolated lingual dystonia and oromandibular dystonia affecting the tongue. J Neurol 2016;263(09):1702-1708 
50. Baylor C, Yorkston K, Eadie T, Kim J, Chung H, Amtmann D. The Communicative Participation Item Bank (CPIB): item bank calibration and development of a disorder-generic short form. J Speech Lang Hear Res 2013;56(04):1190-1208

51. Hogikyan ND, Sethuraman G. Validation of an instrument to measure voice-related quality of life (V-RQOL). J Voice 1999;13(04):557-569
52. Jacobson BH, Johnson A, Grywalski C, Silbergliet A, Jacobson G, Benninger MS. Voice Handicap Index (VHI): development and validation. Am J Speech Lang Pathol 1997;6(03):66-69

53. Piacentini V, Zuin A, Cattaneo D, Schindler A. Reliability and validity of an instrument to measure quality of life in the dysarthric speaker. Folia Phoniatr Logop 2011;63(06):289-295 